\title{
Nonlinear low-frequency wave aspect of foreshock density holes
}

\author{
N. Lin ${ }^{1}$, E. Lee ${ }^{1}$, F. Mozer ${ }^{1}$, G. K. Parks ${ }^{1}$, M. Wilber ${ }^{1}$, and H. Rème ${ }^{2}$ \\ ${ }^{1}$ Space Sciences Laboratory, UC Berkeley, Berkeley, CA 94720, USA \\ ${ }^{2}$ Centre d'Etude Spatiale des Rayonnement, Paul Sabatier University, Toulouse, France
}

Received: 6 May 2008 - Revised: 13 October 2008 - Accepted: 13 October 2008 - Published: 25 November 2008

\begin{abstract}
Recent observations have uncovered shortduration density holes in the Earth's foreshock region. There is evidence that the formation of density holes involves nonlinear growth of fluctuations in the magnetic field and plasma density, which results in shock-like boundaries followed by a decrease in both density and magnetic field. In this study we examine in detail a few such events focusing on their low frequency wave characteristics. The propagation properties of the waves are studied using Cluster's four point observations. We found that while these density hole-structures were convected with the solar wind, in the plasma rest frame they propagated obliquely and mostly sunward. The wave amplitude grows non-linearly in the process, and the waves are circularly or elliptically polarized in the left hand sense. The phase velocities calculated from four spacecraft timing analysis are compared with the velocity estimated from $\delta E / \delta B$. Their agreement justifies the plane electromagnetic wave nature of the structures. Plasma conditions are found to favor firehose instabilities. Oblique Alfvén firehose instability is suggested as a possible energy source for the wave growth. Resonant interaction between ions at certain energy and the waves could reduce the ion temperature anisotropy and thus the free energy, thereby playing a stabilizing role.
\end{abstract}

Keywords. Interplanetary physics (Solar wind plasma) Space plasma physics (Nonlinear phenomena; Waves and instabilities)

\section{Introduction}

Earth's bow shock converts incident solar wind into shocked magnetosheath plasma, and provides a natural laboratory for a variety of plasma processes. It has been known that the foreshock region of the Earth's quasi-parallel bow shock is

Correspondence to: N. Lin

(nlin@ssl.berkeley.edu) rich in plasma waves and disturbances. Various types of waves with frequencies ranging from $\mathrm{mHz}$ to $\mathrm{kHz}$ have been observed there. There have been numerous studies on the waves occurring in the foreshock region (cf. Russell and Hope, 1983; Greenstadt et al., 1995; Burgess, 1997; Wilkinson, 2003; Eastwood et al., 2005). Among the disturbances in the foreshock region, there are several types of transient perturbations which may develop large amplitude variations of the magnetic and electric field, and the plasma density. Transient structures characterized by short durations $(\sim 10 \mathrm{~s})$ and large amplitude fluctuations of field and plasma parameters have often been observed. Examples of such structures include short large-amplitude magnetic structures (SLAMS) (Schwartz et al., 1992; Lucek et al., 2002; Stasiewicz et al., 2003), less commonly observed hot flow anomalies (HFAs) (Schwartz et al., 1985, 1991; Paschman et al., 1988; Onsager et al., 1990; Sibeck et al., 1998), hot diamagnetic cavities (HDCs) (Thomsen et al., 1986; Lucek et al., 2004). The development of these structures that evolved out of the upstream wave field might play some role in the solar windmagnetosheath transition (Sibeck et al., 2002).

Recently, Parks et al. (2006, 2007) reported observations of short-duration (4-90s) foreshock density holes. The strong depletions in density, which can be as low as 0.01 of the ambient solar wind density, had not been reported in earlier studies of transient structures. Density holes seem to be embedded within solar wind current sheets of moderate shear, and can grow rapidly. They are filled with hot particle populations, which presumably can account for an overpressure driving the expansion when it is observed. The upstream edges of density holes are often seen as a sharp enhancement of magnetic field. An association of density holes with strong upstream pulsations has been noted previously (Thomsen et al., 1990; Behlke et al., 2003). Density holes also share some characteristics with HFAs and HDCs, typically including significant bulk flow deflections, hot interior plasmas, and enhanced edge densities and magnetic fields. Durations and

Published by Copernicus Publications on behalf of the European Geosciences Union. 

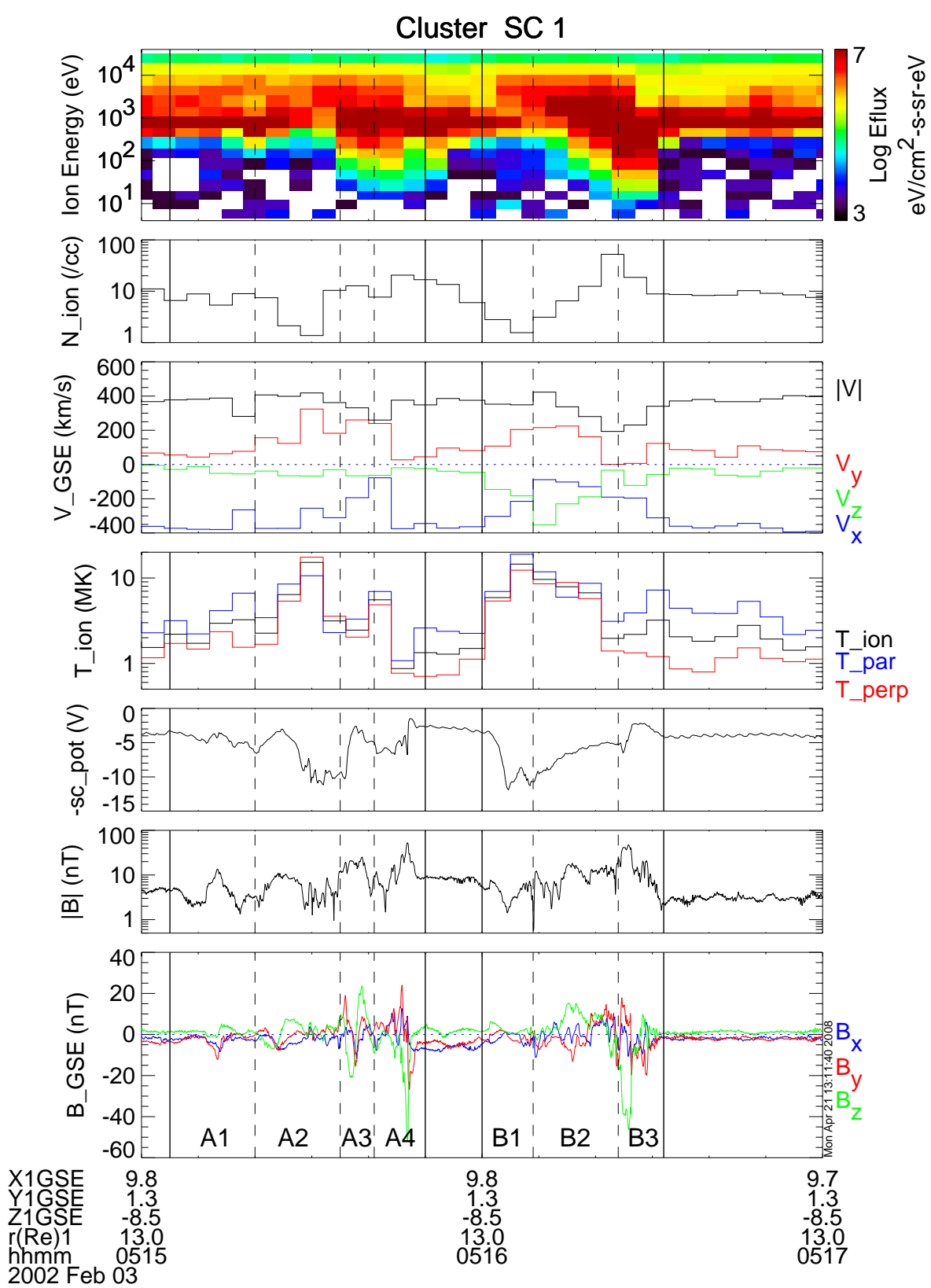

Fig. 1a. Observations of Cluster 1 on 3 February 2002, 05:15-05:17 UT. From top to bottom: (1) Energy spectra of ion flux from CIS-HIA instrument; (2) The ion density; (3) Velocity components in GSE coordinates: $\mathrm{V}_{x}$ (blue), $V_{y}$ (red), $V_{z}$ (green) and the total velocity (black); (4) The ion temperature (black) overplotted with temperature components in directions parallel (blue) and perpendicular (red) to the magnetic field; (5) Negative spacecraft potential as a proxy for the electron density; (6) The magnetic field strength; (7) Magnetic field components in GSE coordinates: $B_{x}$ (blue), $B_{y}$ (red), $B_{z}$ (green).

transverse scales for density holes are much shorter, and the minimum densities are often much lower, however.

There is evidence that the formation of density holes likely involves the development of nonlinear waves, which have not been well studied, and for which there is no complete theory. This report presents detailed analyses of density holes, which reveals the low frequency electro-magnetic wave nature of the structures, and their non-linear growth which forms shock-like edges of the structures.

\section{Observations and analysis}

Three density hole structures are observed between 05:15 and $05: 17$ UT on 3 February 2002, by Cluster spacecraft, at $\sim(9.76,1.27,-8.46) R_{E}$ in GSE coordinates, upstream of the bow shock. Figure 1a shows plasma and magnetic field observations performed by Cluster Ion Spectrometer (CIS) (Rème et al., 1997) at $4 \mathrm{~s}$ per sample and Flux Gate Magnetometer (Balogh et al., 1997) experiments on board 

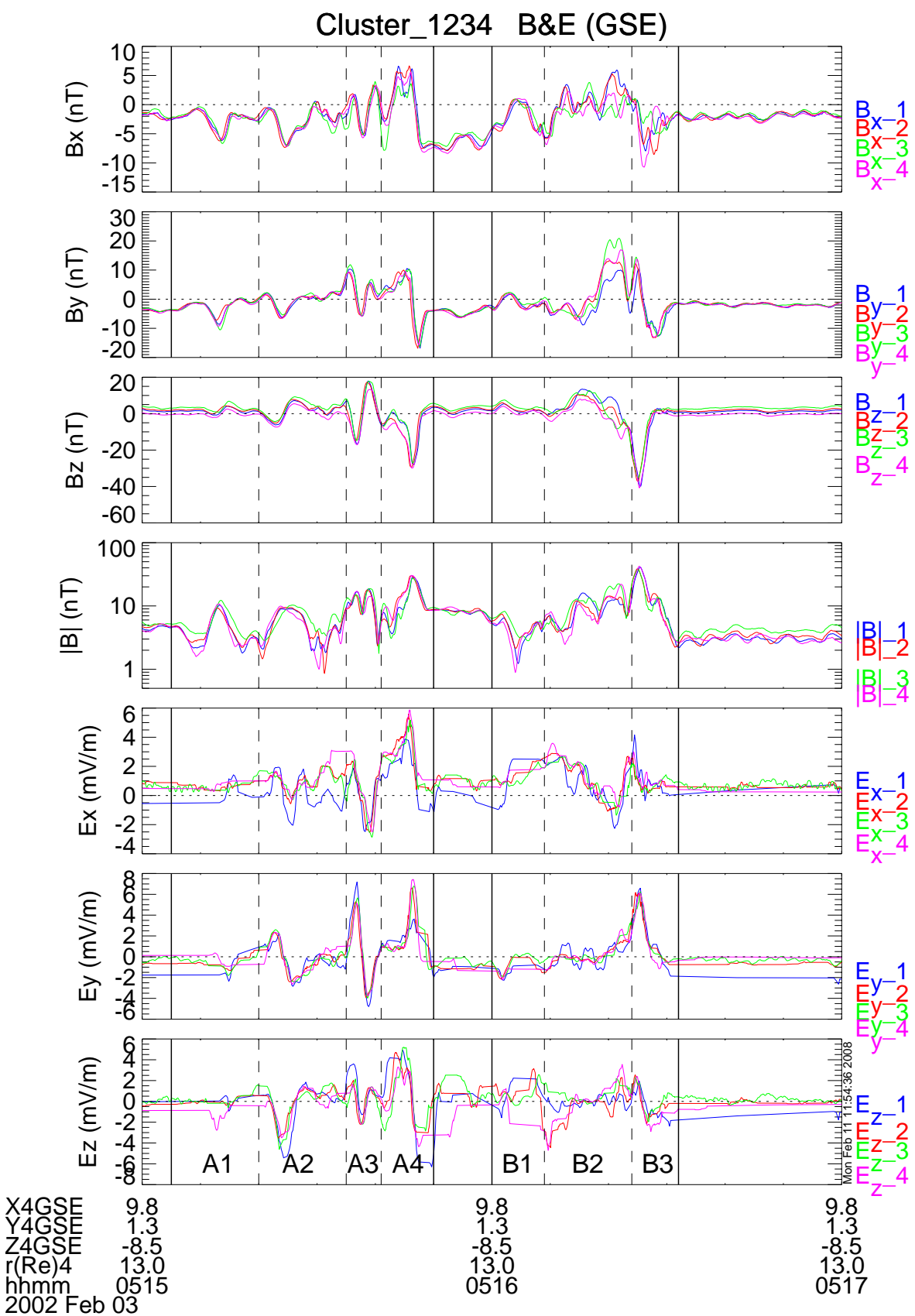

Fig. 1b. Smoothed magnetic and electric field data produced by applying a running average with a window of $1.5 \mathrm{~s}$.

Cluster 1. The spacecraft, after crossing the bow shock around $\sim 05: 14: 40 \mathrm{UT}$ at a distance of $\sim 12.98 R_{E}$ from the Earth, was in the Earth's foreshock region where back streaming ions with an energy of several $\mathrm{keV}$ are observed in addition to the $\sim 1 \mathrm{keV}$ solar wind ions. The spacecraft potential with a resolution of 5 samples per second, obtained from electric field measurements (Gustafsson et al., 2001) is displayed in panel 6 as a proxy for the electron density.

Two density holes in a row are observed between 05:15:20 and 05:15:50 UT (subintervals A2, A3, and A4), and another hole is seen between 05:16:00 and 05:16:32 UT (subintervals B1, B2, and B3). We have marked the two periods as A and $B$, with A divided into four subintervals (A1 to A4, separated by dashed lines), and $\mathrm{B}$ divided into three subintervals (B1 to B3). As we can see later from the wave hodograms, each of the subintervals, except B2, contains approximately a full cycle of the wave. The decrease in density is obvious as the negative spacecraft potential decreases. Measurements from CIS (panel 2) show that the spin-averaged density in the holes decreases to as low as $\sim 1 \mathrm{~cm}^{-3}$ from $\sim 10 \mathrm{~cm}^{-3}$ 
in the solar wind (between 05:16:50 and 05:17:00). In addition to the drop in the density, these structures exhibit other characteristics typical of density holes. The velocity is deflected within the holes, from mainly in the $\mathrm{x}$-direction in the solar wind to a direction with significant $\mathrm{y}$ and $\mathrm{z}$ components inside the holes (panel 3). The $(<40 \mathrm{keV})$ ion temperature has increased to $\sim 10 \mathrm{MK}$ inside the holes from $\sim 2 \mathrm{MK}$ in the solar wind (see panels 4 and 1). The magnetic field strength (shown in panel 6 in a log scale) shows enhancements in the upstream edges of the holes, coincident with increases in the plasma density, and slight decreases (with fluctuations) with the drop of density. While it is not uncommon to see highly correlated in-phase variations between the density and magnetic field magnitude of density-hole structures, in these events the variations of magnetic field and the density are weakly in-phase correlated.

The above density holes are observed by all four Cluster spacecraft, which are separated by less than $\sim 200 \mathrm{~km}$ during the event. The simultaneous observations from four spacecraft have allowed us to determine propagation properties of a wave, which include the direction of the wave normal, the phase velocity in both the spacecraft frame and in the plasma rest frame, and the polarization of the wave. Below we will present properties of the variations of the magnetic and electric field of the density-holes, from the beginning of small amplitude fluctuations to fully developed shock-like upstream edges of the structure.

\subsection{Propagation of the wave}

The phase velocity and propagation direction of the waves are calculated using timing analysis from four spacecraft observations, under the assumptions that the wave front is planar and uniform on the scale of the spacecraft separation and that the waves are propagating at a constant phase velocity. If spacecraft $\beta$ located at $\boldsymbol{R}_{\beta}$ observes the phase front at time $t_{\beta}$, then the propagation direction of a plane wave $\hat{\boldsymbol{e}}_{k}$ and the phase velocity $V_{\varphi}$ in the spacecraft frame can be calculated by solving the equations $\left(\boldsymbol{R}_{\beta}-\boldsymbol{R}_{1}\right) \bullet \hat{\boldsymbol{e}}_{k}=V_{\varphi} \cdot\left(t_{\beta}-t_{1}\right)$ and $V_{\varphi}=\boldsymbol{V}_{\varphi} \bullet \hat{\boldsymbol{e}}_{k}$, where $\beta=2,3,4$. Timing lags between the spacecraft are determined using cross-correlation maxima after smoothing the data to remove high-frequency fluctuations. Under the above assumptions, the unit vector of the phase speed, $\hat{\boldsymbol{e}}_{k}$, obtained by this method is actually in the direction of the wave vector, $\boldsymbol{k}$. This method (Schwartz, 2000; Eastwood et al., 2002, 2003) has been well-tested in numerous Cluster studies to date. The phase velocity in the plasma rest frame can then be calculated by subtracting the solar wind velocity in the propagation direction of the waves.

To apply the method, we first low-pass filter the magnetic field data by applying a running average with a window of $1.5 \mathrm{~s}$ to remove high frequency fluctuations. The smoothed magnetic field variations for four spacecraft are shown in the first four panels of Fig. 1b. The variations of the field at four spacecraft are well correlated. In the intervals where the density holes are observed, the magnetic field fluctuations contain both compressional and transverse components. The peak to peak amplitude can be as large as $40 \mathrm{nT}$, much larger than the ambient magnetic field ( $\sim 5 \mathrm{nT})$, indicating development of non-linear fluctuations. Since the magnetic field is unstable and the wave normal is likely to vary with time, we will apply the analysis to each subinterval in Fig. 1.

As an example, we examine interval A3 (05:15:3505:15:41 UT), when the fluctuations have developed a shock like edge after a drop in density and in magnetic field strength during A2. The timing analysis yields a unit wave normal $\hat{\boldsymbol{e}}_{k}=(-0.89,0.43,-0.18)$ and the observed phase velocity, $V_{\text {obs }}$, of $281.5 \mathrm{~km} \mathrm{~s}^{-1}$. This means that the observed wave front propagates mainly anti-sunward in the spacecraft frame. Using the ambient solar wind velocity measured during a period before the hole between 05:15:50 and 05:15:55 UT, $\boldsymbol{V}_{s w}=(-350,70,-20) \mathrm{km} \mathrm{s}^{-1}$ in GSE coordinates, we may calculate the phase velocity of the wave in the plasma rest frame in the direction of $\hat{\boldsymbol{e}}_{k}$ as $V_{0}=V_{\text {obs }}-\boldsymbol{V}_{s w} \bullet \hat{\boldsymbol{e}}_{k}=-62 \mathrm{~km} \mathrm{~s}^{-1}$. The minus sign indicates that in the plasma frame the wave front propagates in the direction opposite to the observed phase velocity. Thus the wave propagates nearly sunward at a velocity of $62 \mathrm{~km} \mathrm{~s}^{-1}$ in the plasma, but its convection towards the Earth results in an observed phase velocity of $281.5 \mathrm{~km} \mathrm{~s}^{-1}$. The plasma frame velocity is greater than Alfvén velocity $\left(\sim 47 \mathrm{~km} \mathrm{~s}^{-1}\right)$ in the ambient solar wind, where $B \sim 8.3 \mathrm{nT}$ and a plasma density of $n \sim 15 \mathrm{~cm}^{-3}$ (taken from electron measurements). The observed $\hat{\boldsymbol{e}}_{k}$ vector constitutes a large angle, $\theta_{B k}$, with the ambient magnetic field. In this case, $\theta_{B k}$ ranges between $60^{\circ}$ and $68^{\circ}$ at the four spacecraft. It should be noted that there is a significant uncertainty in defining the "ambient magnetic field" for the fluctuations, since the fluctuations are non-linear with large amplitudes, the "background field" during the fluctuations is also varying with time at the wave frequency. Here we took the average of magnetic field measured in a short period right before an event, when the solar wind seems to be rather "stable", as the solar wind field. For event $\mathrm{A}$, this period is between 05:15:50 and 05:15:55 UT, while for event $\mathrm{B}$, this period is between $05: 16: 32-05: 16: 42 \mathrm{UT}$.

\subsection{The wave polarization}

Since we have determined the wave normal, we may project the magnetic field onto the plane of the wave front, which is perpendicular to the wave normal, and resolve the projected field into two orthogonal components on the plane. The unit vector of the first component is taken as $\boldsymbol{t}_{1}=\hat{\boldsymbol{e}}_{k} \times \boldsymbol{b}_{0} /\left|\hat{\boldsymbol{e}}_{k} \times \boldsymbol{b}_{0}\right|$, where $\boldsymbol{b}_{0}$ is the unit vector of the background magnetic field defined above, while the second component is $\boldsymbol{t}_{2}=\hat{\boldsymbol{e}}_{k}$ $\times t_{1} /\left|\hat{\boldsymbol{e}}_{k} \times \boldsymbol{t}_{1}\right|$. By plotting the hodogram of the two components we are able to observe the polarization of the wave. We have projected the magnetic field for the interval onto the two orthogonal components which are perpendicular to the wave normal, and plotted the hodogram in Fig. 2 (left) for 
Table 1. Observed polarization senses are the results at four spacecraft, respectively.

\begin{tabular}{|c|c|c|c|c|c|c|c|c|}
\hline & Interval & $\hat{\boldsymbol{e}}_{k(x, y, z)}$ & $\begin{array}{c}V_{\mathrm{obs}} \\
\mathrm{Km} \mathrm{s}^{-1}\end{array}$ & $\begin{array}{c}\delta E / \delta B \\
\mathrm{Km} \mathrm{s}^{-1}\end{array}$ & $\begin{array}{c}V_{s w}(x, y, z) \\
\mathrm{Km} \mathrm{s}^{-1}\end{array}$ & $\begin{array}{c}V_{0} \\
\mathrm{Km} \mathrm{s}^{-1}\end{array}$ & $\begin{array}{c}\theta_{B k}(\text { deg. }) \\
\left(\theta_{1}, \theta_{2}, \theta_{3}, \theta_{4}\right)\end{array}$ & Polarization \\
\hline $\mathrm{A} 1$ & $05: 15: 05-05: 15: 20$ & $-0.80,0.35,-0.48$ & 245.5 & $261 \pm 64$ & $-350,70,-20$ & -70.1 & $66,70,76,59$ & $\mathrm{R}, \mathrm{R}, \mathrm{R}, \mathrm{R}$ \\
\hline $\mathrm{A} 2$ & $05: 15: 20-05: 15: 35$ & $-0.94,0.33,-0.09$ & 272.9 & $462 \pm 46$ & $-350,70,-20$ & -80.9 & $54,56,60,53$ & $\mathrm{R}, \mathrm{R}, \mathrm{R}, \mathrm{R}$ \\
\hline A3 & $05: 15: 35-05: 15: 41$ & $-0.89,0.43,-0.18$ & 281.5 & $293 \pm 22$ & $-350,70,-20$ & -62.0 & $61,64,68,60$ & $\mathrm{R}, \mathrm{R}, \mathrm{R}, \mathrm{R}$ \\
\hline A4 & $05: 15: 41-05: 15: 50$ & $-0.93,0.32,-0.18$ & 201.1 & $250 \pm 23$ & $-350,70,-20$ & -150.6 & $55,57,62,53$ & $\mathrm{R}, \mathrm{R}, \mathrm{R}, \mathrm{R}$ \\
\hline A & $05: 15: 05-05: 15: 50$ & $-0.95,0.23,-0.18$ & 224.2 & - & $-350,70,-20$ & -129.3 & $52,55,60,50$ & \\
\hline B1 & $05: 16: 00-05: 16: 09$ & $-0.96,0.27,-0.06$ & 188.1 & $552 \pm 43$ & $-370,100,-40$ & -196.8 & $57,61,72,53$ & $\mathrm{R}, \mathrm{R}, \mathrm{R}, \mathrm{R}$ \\
\hline $\mathrm{B} 2$ & 05:16:09-05:16:24 & $-0.82,0.58,0.02$ & 148.9 & $?$ & $-370,100,-40$ & -209.9 & $74,73,80,73$ & $\sim \mathrm{R}$ \\
\hline B3 & $05: 16: 24-05: 16: 32$ & $-0.96,0.23,0.17$ & 154.3 & $171 \pm 14$ & $-370,100,-40$ & -216.1 & $50,50,59,55$ & $\mathrm{R}, \mathrm{R}, \mathrm{R}, \mathrm{R}$ \\
\hline $\mathrm{B}$ & 0516:00-0516:32 & $-0.92,0.37,0.13$ & 158.8 & - & $-370,100,-40$ & -213.2 & $59,59,66,62$ & \\
\hline
\end{tabular}

$V_{0}=V_{\mathrm{obs}}-\hat{\boldsymbol{e}}_{k} \boldsymbol{V}_{s w}$ is the wave phase velocity in the plasma frame in the direction of $\hat{\boldsymbol{e}}_{k}$.

$\theta_{B k}$ is the angle between the $\boldsymbol{k}$ vector and the magnetic field of the solar wind upstream of the structure observed at Cluster $1\left(\theta_{1}\right), 2\left(\theta_{2}\right), 3$ $\left(\theta_{3}\right), 4\left(\theta_{4}\right)$.

$\delta E / \delta B$ is the average of the values obtained from four spacecraft.

spacecraft 1 . The wave observed at all four spacecraft is elliptically (with a rather small eccentricity) polarized in right handed sense. Since the wave propagates in the sunward direction in the plasma rest frame and convected toward the spacecraft by the solar wind, the observed right hand polarization thus implies a left hand polarization in the plasma frame, which indicates that the wave is Alfvén wave.

The polarization of the electric field for the same interval has also been examined in the same way. The electric field is first running averaged over $1.5 \mathrm{~s}$ (shown in the last three panels of Fig. 1b) and then projected onto the same two orthogonal components (Fig. 2, right). Electric field measurements of Cluster are made on the spin plane only, the third component of the electric field $E_{z}$ is calculated by assuming that $\boldsymbol{E} \cdot \boldsymbol{B}=0$. This may introduce a large error when $\left|B_{x} / B_{z}\right|$ or $\left|B_{y} / B_{z}\right|$ is large. Therefore, we consider the calculated $E_{z}$ valid only when $\left|B_{x} / B_{z}\right|$ and $\left|B_{y} / B_{z}\right|$ are less than 5 . This restriction has produced a number of data gaps in the electric field data plotted in Fig. 1b, which has been interpolated with straight lines. Fortunately the calculated electric field data are reasonably good for this study, especially in key periods when the wave amplitude grew significantly.

The hodogram of the electric field at four spacecraft also shows right hand elliptical polarization, consistent with that of the magnetic field. We also notice that the major axes of the ellipse in the magnetic field hodograms are basically perpendicular to those in the corresponding hodograms of the electric field, a feature consistent with the properties of a plane electromagnetic wave.

From the hodograms the ratio of the amplitude of the electric field variations to that of the magnetic field, $\delta E / \delta B$, can be obtained, and is used as an estimation of the phase velocity of the wave. For this interval, $\delta E / \delta B$ measured at four spacecraft are $328.9,289.2,281.3,272.4 \mathrm{~km} \mathrm{~s}^{-1}$, separately, which re-

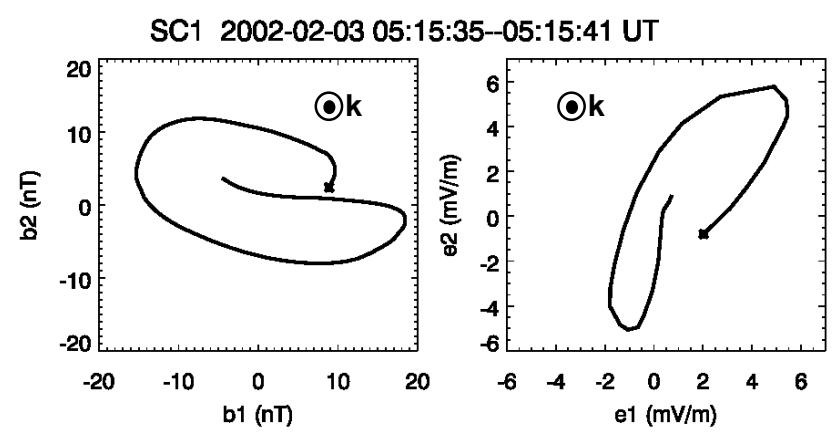

Fig. 2. Hodograms of the magnetic field (left) and electric field (right) for interval A3, which show right hand polarization with respect to the $\boldsymbol{k}$ vector (pointing out of the paper) in the spacecraft frame. The star signs are the starting points of the curves.

sults in an average of $293 \pm 22 \mathrm{~km} \mathrm{~s}^{-1}$, in a reasonably good agreement with the observed phase velocity determined by the timing analysis. The agreement seems to justify the plane wave assumption in the timing analyses and reveal the electromagnetic wave nature of the fluctuations. The wavelength of the wave is estimated as $\sim 1700 \mathrm{~km}$, much larger than the spacecraft separations, which also justifies the assumption of uniform wave front on the scale of the spacecraft separation.

The same analysis has been applied to the intervals A1 to A4 and B1 to B3 in Fig. 1, and the results are listed in Table 1.

\subsection{Growth of the wave}

The results in Table 1 show that for most of the subintervals, each of which roughly corresponds to a cycle of the wave phase variation (as shown later in Fig. 3), the wave properties are similar to those for interval A3, which we analyzed in detail above. The observed $\hat{\boldsymbol{e}}_{k}$ vectors are mainly pointing 
SC1 2002-02-03 05:15:05--05:15:50 UT
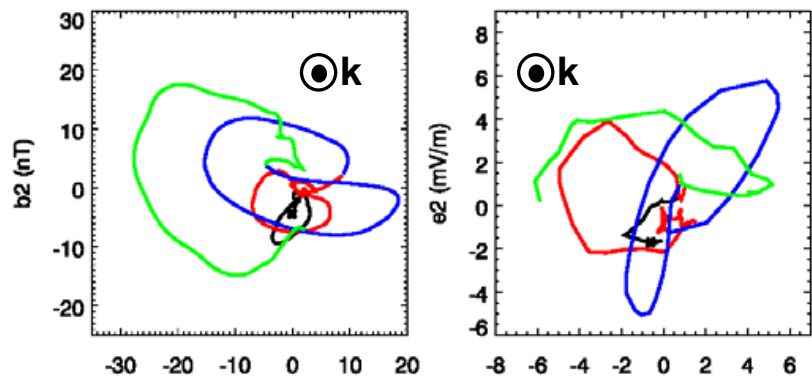

SC2 2002-02-03 05:15:4.59--05:15:49.5 UT
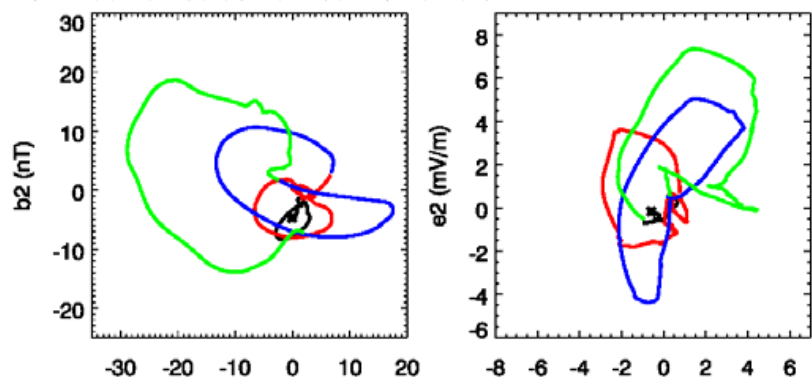

SC3 2002-02-03 05:15:5.00-05:15:50.0 UT
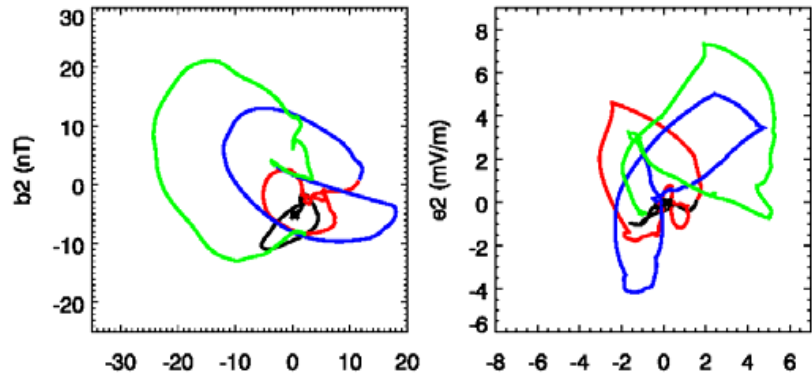

SC4 2002-02-03 05:15:4.82--05:15:49.8 UT
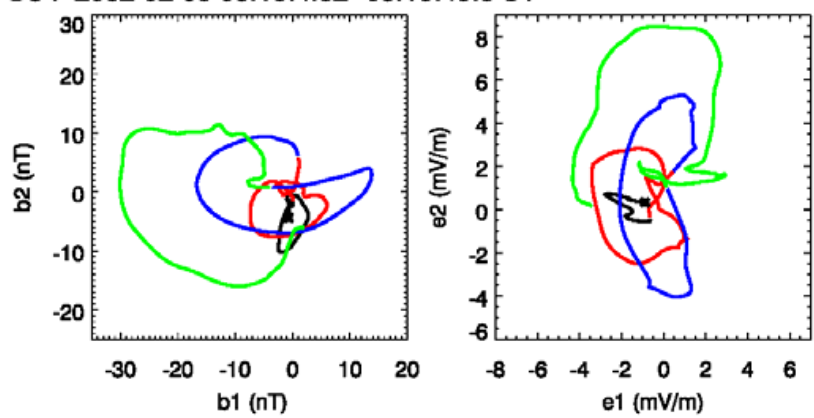

Fig. 3a. Hodogram for the interval A. Left panels: polarization of the magnetic waves observed at four Cluster spacecraft. Right panels: polarization of the electric waves. The $\boldsymbol{k}$ vector is pointing out of the paper.

anti-sunward. In the spacecraft frame the waves propagate towards the earth at a phase velocity of $150-300 \mathrm{~km} \mathrm{~s}^{-1}$, at oblique angles of $\sim 50^{\circ}-70^{\circ}$ to the ambient magnetic field. The observed polarization is right handed. In the plasma rest frame, these waves propagate in the sunward direction, thus the waves must be polarized in the sense opposite to that in the spacecraft frame. The values of $\delta E / \delta B$ are in reasonable
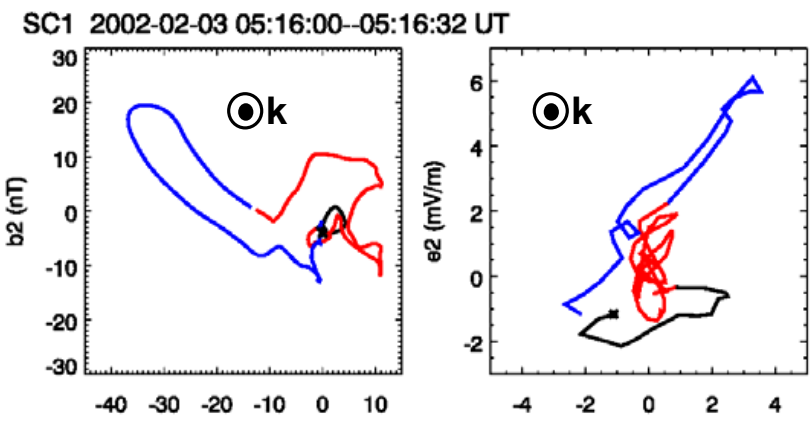

SC2 2002-02-03 05:15:59.5--05:16:31.5 UT
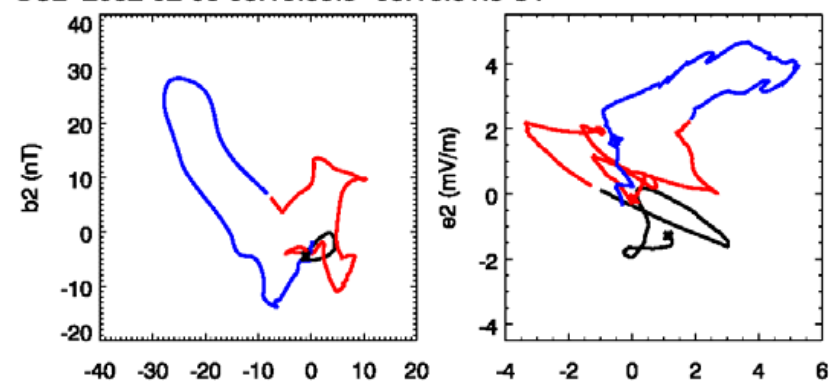

SC3 2002-02-03 05:15:59.7--05:16:31.7 UT
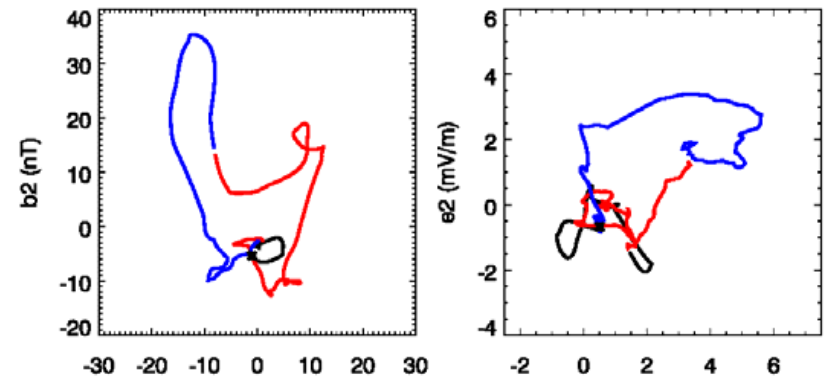

SC4 2002-02-03 05:15:59.7--05:16:31.7 UT
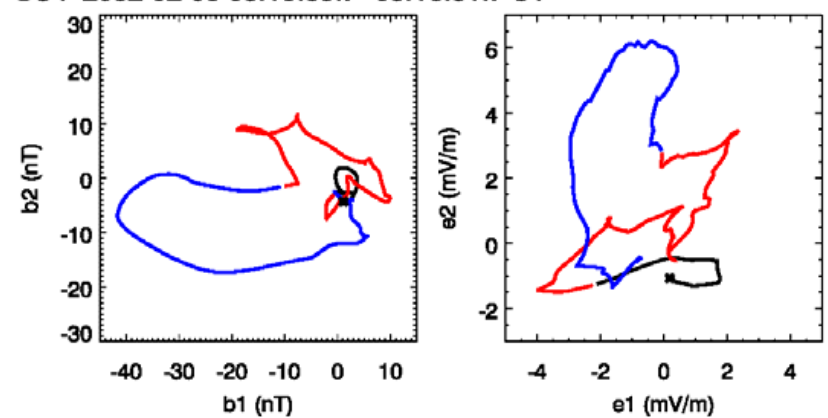

Fig. 3b. The same as Fig. 3a but for interval B.

agreement with the observed phase velocity, $V_{\mathrm{obs}}$, for most subintervals, except for $\mathrm{A} 2$ and $\mathrm{B} 1$, where $\delta E / \delta B$ is two to three times larger than $V_{\text {obs }}$. During interval B2, the magnetic and electric field fluctuations were much more irregular such that $\delta E / \delta B$ could not be determined. Judged from the magnetic field measurements (seen in Fig. 3b) the polarization for this period is roughly right-handed in the spacecraft frame.

Since the direction of the wave vectors in subintervals are similar, we apply the timing analysis to a period combining 

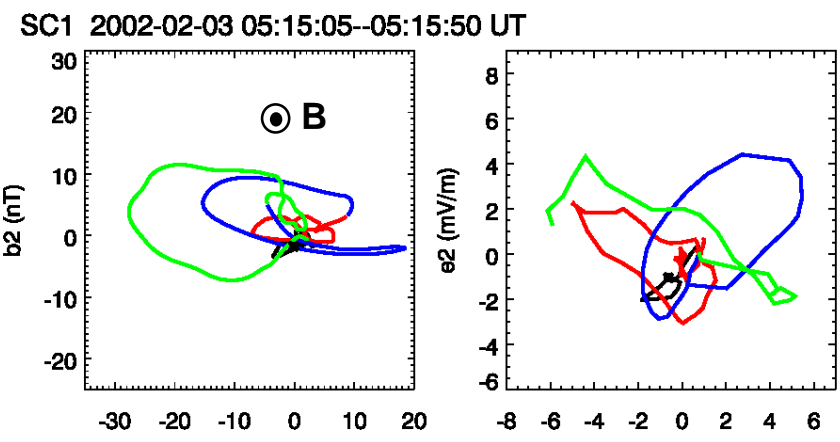

SC3 2002-02-03 05:15:5.00--05:15:50.0 UT
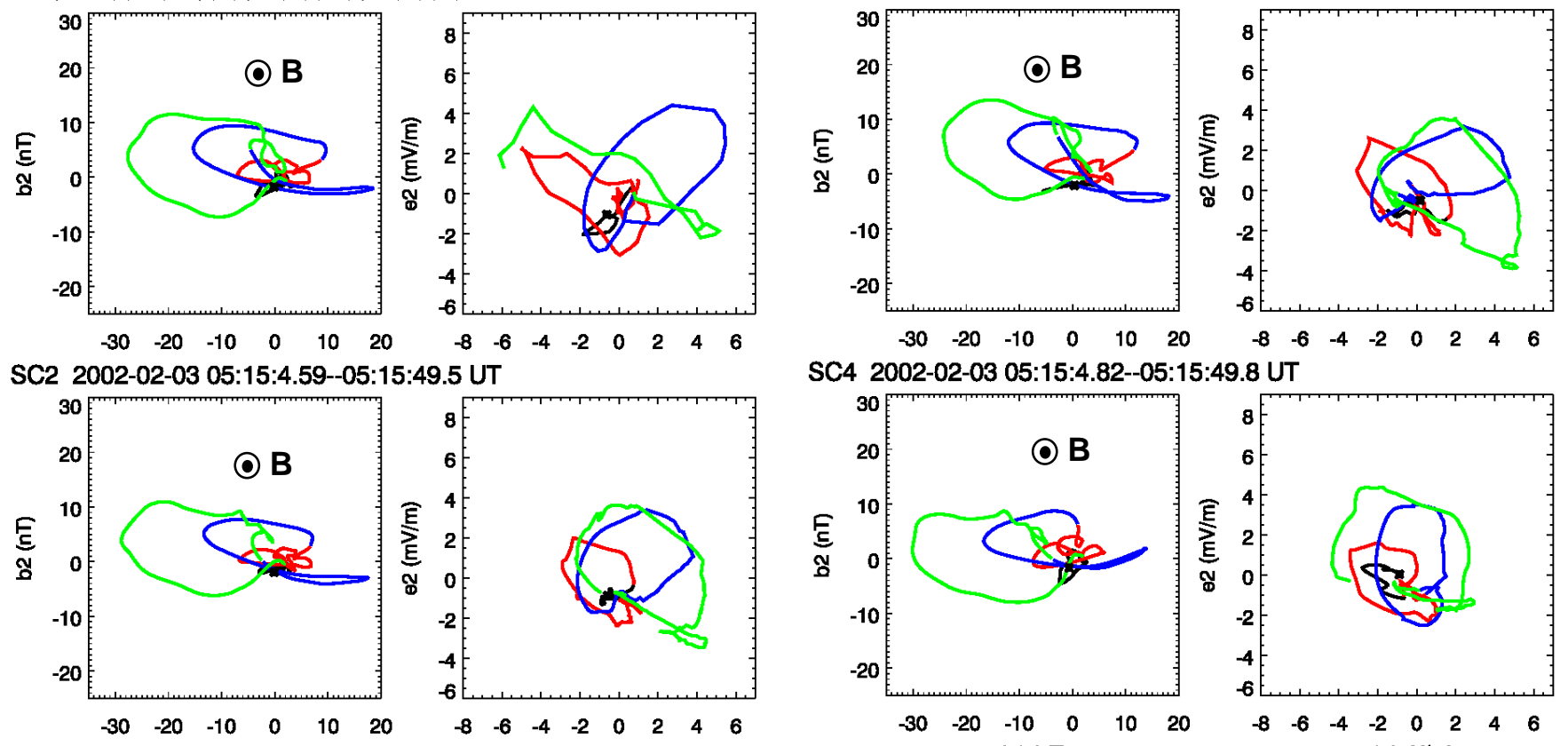

SC4 2002-02-03 05:15:4.82--05:15:49.8 UT
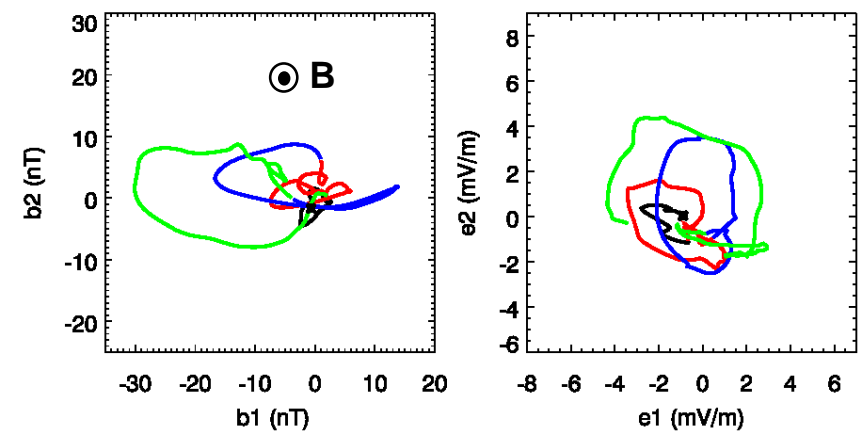

Fig. 4. Polarization of the waves with respect to the ambient magnetic field for interval A, plotted in the same format as that of Fig. 3. The magnetic field is out of the paper.

A1 to A4, and a period combining B1 to B3, respectively. The results are listed in Table 1 as periods $\mathrm{A}$ and $\mathrm{B}$, which can be considered as the "average" properties of the waves in each period. In each period we saw the development and growth of the low frequency fluctuations to the shocklike edge of a density-hole structure. We plotted the continuous hodogram for period A and B in Fig. $3 \mathrm{a}$ and b. In Fig. 3a, the hodograms for subintervals A1 to A4 are plotted in black-red-blue-green, respectively. The figure shows that both magnetic and electric field vectors continuously rotate in right hand sense with respect to $\hat{\boldsymbol{e}}_{k}$ for four cycles, each of which corresponds to a subinterval. A striking feature is that in the process the wave amplitude is growing gradually but significantly. For example, at Cluster 1, the magnetic field amplitude increases from $\sim 5 \mathrm{nT}$ in A1 to $\sim 20 \mathrm{nT}$ in A4, which means the wave grows four times bigger in $45 \mathrm{~s}$. It is likely that what we observed was the growing of the wave amplitude with time, although there is another possibility that we were seeing a spatially varying magnetic field structure convecting past the spacecraft. For the latter possibility to occur, however, the structure would have had flux ropetype helical magnetic field with a smaller "cross section" at the down stream end and a much larger "cross section" at the upstream end. This structure would have been formed by an unknown mechanism in the solar wind and propagated sunward in the plasma frame, but was convected earthward by the solar wind. Such structure would have a large scale size of $\sim 10000 \mathrm{~km}$, considering the observed velocity of $\sim 220 \mathrm{~km} \mathrm{~s}^{-1}$ and duration of $\sim 45 \mathrm{~s}$ (for period A). In this study we adopt the view that it is more natural to interpret the observed phenomena as resulting from time-development.

For period B (Fig. 3b), the hodograms of three subintervals are plotted in black, red and blue sequentially. The magnetic field variations in the first interval $\mathrm{B} 1$ show a nice right hand polarization with a small amplitude $(\sim 2-3 \mathrm{nT})$, while in B3, the wave still polarized in right hand sense at a much larger amplitude $(\sim 50 \mathrm{nT})$. During the second interval B2, the variations are more irregular, especially in electric field measurements, that we cannot determine the polarization and the ratio $\delta E / \delta B$, but the rotation of the magnetic field shows basically in the right handed sense. The intervals A3, A4, and $\mathrm{B} 3$ contain shock-like edges of density holes, the amplitude of the waves increases significantly during these subintervals suggest that the shock-like edges in the upstream side of density holes, which form a structure similar to SLAMS, may have been developed from a nonlinear growth of ULF waves.

So far we have been describing the wave polarization as the rotational sense of the wave electric and magnetic field with respect to the observed unit wave normal $\hat{\boldsymbol{e}}_{k}$, since $\hat{\boldsymbol{e}}_{k}$ is the quantity that can be determined with four spacecraft measurements. In a magnetized plasma, it is important to know the polarization of the wave with respect to the ambient magnetic field, especially when considering the interaction between charged particles and the waves. In our case, as mentioned above, the angle between observed $\hat{\boldsymbol{e}}_{k}$ and the 
solar wind magnetic field is $\sim 60^{\circ}$. Thus the polarization of the waves with respect to the solar wind magnetic field remains right handed in the spacecraft frame, and is left hand polarized in the solar wind frame. In Fig. 4 we display the hodogram showing the polarization of the waves with respect to the magnetic field for period A. The polarization remains the same but with somewhat different apparent eccentricity.

We notice that in intervals A4 and B3, when the wave amplitude has grown large, the phase velocity $V_{0}$ is faster than the fast mode speed, $V_{\text {fast }} \sim\left(V_{s}^{2}+V_{A}^{2}\right)^{1 / 2}$, where $V_{s}$ is the sound speed and $V_{A}$ is the Alfvén speed. For A3 and A4, $V_{\text {fast }} \sim 115 \mathrm{~km} \mathrm{~s}^{-1}$, taking the magnetic field in the solar wind $B_{s w} \sim 8.3 \mathrm{nT}, T_{e} \sim T_{i} \sim 0.2 \mathrm{MK}$, and the plasma density $n_{s w} \sim 15 \mathrm{~cm}^{-3}$, while for B3, $V_{\text {fast }} \sim 105 \mathrm{~km} \mathrm{~s}^{-1}$ (with the same solar wind conditions but $B_{s w} \sim 2.9 \mathrm{nT}$ ). The phase velocity of the shock like edges of density holes in the plasma frame which exceed the fast mode speed have been reported in previous work (Parks et al., 2006). This suggests that nonlinearly developed fluctuations can propagate much faster than the waves predicted in the linear theory. The wavelength of the waves can be estimated as $l=V_{\mathrm{obs}} \cdot T$. For period A, for Cluster 1, the wavelength is $\sim 1800 \mathrm{~km}$. Here, the wave period, $T$, is taken as $\sim 8 \mathrm{~s}$, the period when the waves have developed to maximum amplitude (i.e. an approximation of the wave period in $\mathrm{A} 3, \mathrm{~A} 4$, and $\mathrm{B} 3$ ), $V_{\mathrm{obs}}=224.2 \mathrm{~km} \mathrm{~s}^{-1}$, from Table 1. For period B, the wavelength is estimated as $\sim 1270 \mathrm{~km}$. These wavelengths are about the gyroradius of $\mathrm{keV}$ protons. The right hand sense of the polarization seems to be common for magnetic field fluctuations in several other density hole events we examined. We notice that the mostly right handed polarization (in the spacecraft frame) is similar to the SLAMS polarization reported previously (Schwartz et al., 1992). This suggests that some of SLAMS could have been the upstream edges of density holes or vice versa (cf. Thomsen et al., 1990; Behlke et al., 2003).

\section{Discussion}

We have presented observations of density holes in the foreshock region and associated low frequency electromagnetic waves. Our analysis shows that the waves are Alfvén mode waves with left hand polarization and propagate nearly sunward in the solar wind frame but are convected earthward toward the spacecraft by the solar wind, and that the spacecraft observed them as right handed polarized waves. The wave amplitudes are growing rapidly and reach a maximum at the upstream edge of the density holes. It may be the case that the overshoots represent the saturation amplitudes for the wave growth, although an alternate interpretation is that compression of the waves through a shock-like transition amplifies the intensity of waves convecting through it.

\subsection{Possible generation mechanism of the waves}

Where is the source of free energy for nonlinear growth of these waves? Although there exist energetic ions reflected back from the bow shock, these ions in the solar wind frame move in the same direction as the waves, with a velocity higher than the phase velocity of the waves. Such ions will not resonate with the wave since the waves are left-handed polarized in the plasma frame.

In Fig. 1a, panel 4, we notice that the parallel temperature, $T_{\|}$, of ions often exceeds the perpendicular temperature, $T_{\perp}$, a condition that may favor the occurrence of firehose instabilities. We have examined the condition for plasma instability to occur during the period under study (cf. Treumann and Baumjohann, 1997; Gary et al., 1998): $\beta_{\|}>\beta_{\perp}+2$, where $\beta_{\|, \perp}=8 \pi \mathrm{nT}_{\|, \perp} / B^{2}$, and $n$ is the ion proton density. It can be written as

$1-T_{\perp} / T_{||}>B^{2} / 4 \pi \mathrm{nT}_{||}$.

In Fig. 5 we display the time variations of plasma $\beta=8 \pi \mathrm{nT} / \mathrm{B}^{2}$ (panel 1) and the criterion for the firehose instability (panel 2), which is calculated as $a=\left(1-T_{\perp} / T_{\|}\right) /\left(B^{2} / 4 \pi \mathrm{nT}_{\|}\right)$. The instability may occur when $a>1$. The results show that the instability condition is satisfied for most of the period, which includes intervals of the solar wind (beyond periods A and B) and subintervals when the wave amplitude is still small (A1 and B1). During the intervals when density minima occur (part of A2, A4 and B2), the instability condition is weak or not satisfied, as the ion temperature becomes more isotropic. We notice that in the upstream side of the period (after B3), the temperature anisotropy of the solar wind is enhanced with $a$ increasing to $\sim 100$.

The existence of electromagnetic firehose instabilities in the solar wind has been investigated previously (e.g. Parker, 1958; Sentman et al., 1981; Yoon, 1990). Most of this earlier work suggested that the instabilities have maximum growth rates along the magnetic field directions. Recent simulation studies (Hellinger and Matsumoto, 2000, 2001; Hellinger and Travnicek, 2006) have revealed that the ion temperature anisotropy, $T_{\|}>T_{\perp}$, may excite an oblique Alfvén firehose instability, in addition to the right hand polarized whistler firehose instability. While the whistler firehose has an overall quasi-linear evolution, the Alfvén firehose has more complicated nonlinear evolution. The Alfvén firehose instability may reach significant amplitudes and heat protons and reduce the anisotropy, then the zero frequency waves are transformed to Alfvén waves. The oblique fire hose can effectively diffuse ions owing to the non-linear evolution. In this study, the waves associated with the density holes and their plasma environments are observed to have many aspects consistent with the occurrence of the oblique Alfvén firehose instability. The parallel fire hose typically dominate over the oblique one except for for high beta plasma. Figure 5 indicates quite large betas in the vicinity of density 

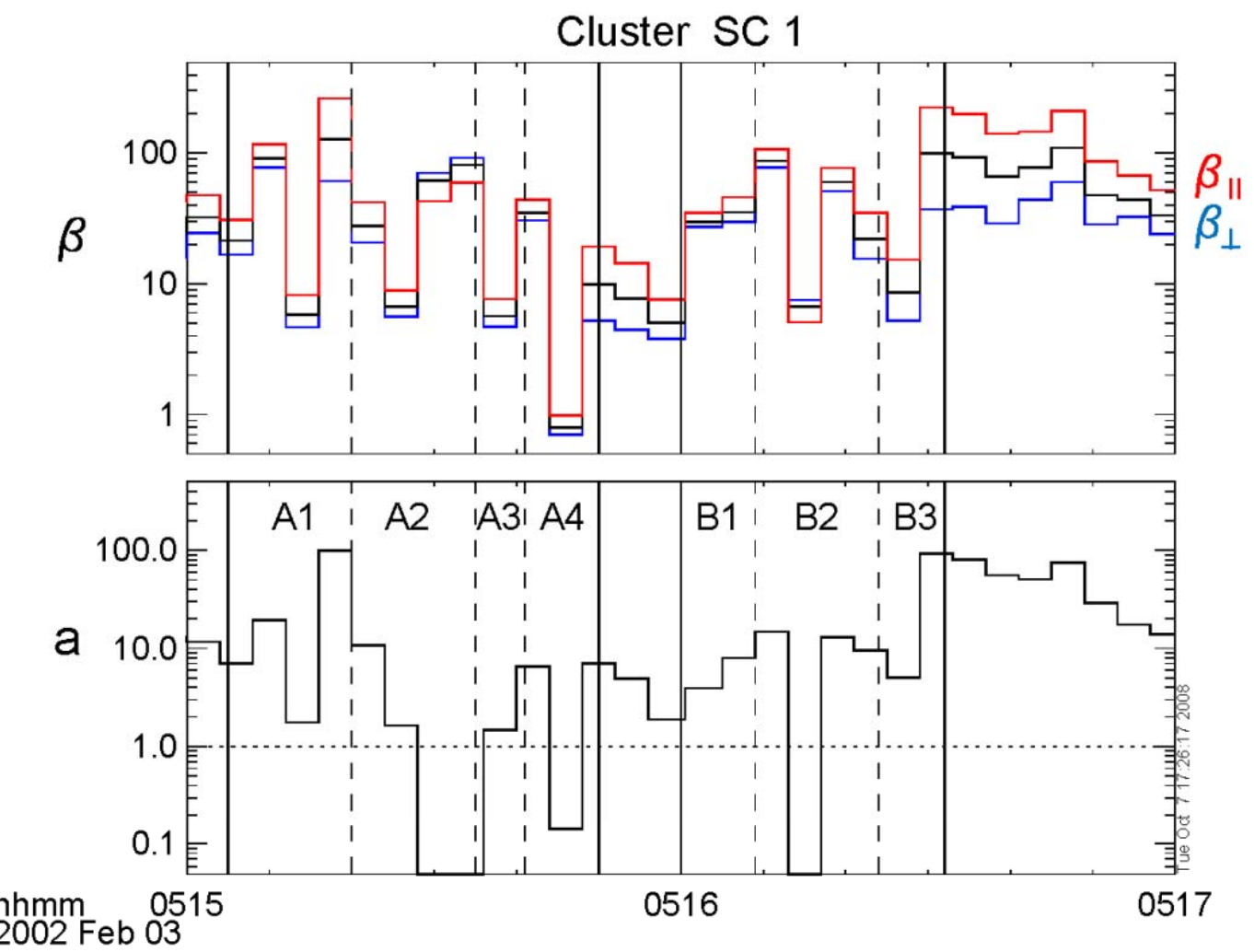

Fig. 5. Time variations of (1) Plasma $\beta$ (black), $\beta_{\|}$(red), and $\beta_{\perp}$ (blue), as defined in the text. (2) Criterion for the firehose instability, $a=\left(1-T_{\perp} / T_{||}\right) /\left(B^{2} / 4 \pi \mathrm{nT}_{||}\right)$.

holes. Comparisons between observations and predictions of the Vlasov linear theory (Hellinger et al., 2006; Kasper et al., 2002) show that the proton temperature anisotropy seems to be constrained by oblique instabilities, including the oblique firehose instability. Figure 5 (second panel) shows that in subintervals when the amplitude of the waves became large (for example, A3, A4 and B3) the anisotropy reduced to a level much lower than that of the solar wind (the interval upstream of B3). This may indicate that the development of the waves reduced the ion anisotropy.

There are other instabilities that could drive oblique Alfvén waves. One that may occur in solar wind conditions is the electromagnetic proton/proton instability (Daughton and Gary, 1998; Daughton et al., 1999). This instability could occur when the proton velocity distribution can be represented as two components (a dense core and a more tenuous beam) of approximately equal temperature with a relative drift velocity parallel to the ambient magnetic field. Such distributions are often observed in the fast solar wind $\left(V>\sim 600 \mathrm{~km} \mathrm{~s}^{-1}\right.$ ) (Daughton et al., 1999). Under the above conditions both magnetosonic and Alfvén waves can be excited. While magnetosonic instabilities have growth rate maximum along the magnetic field direction, Alfvén instabilities have maximum growth at propagation oblique to the field. Results from the simulation study (Daughton and
Gary, 1998) shows that the Alfvén instability may have a low threshold for a large beam density and small core $\beta(\leq 1)$. In our event, the solar wind velocity is $<400 \mathrm{~km} \mathrm{~s}^{-1}$ throughout the interval, and we did not observe clear signatures of a core-beam distribution having two components of approximately equal temperature. Also, the plasma $\beta$ for most of the interval is rather high $(\sim 10-100)$. The behavior and properties of this kind of instabilities in high $\beta$ solar wind are not yet well studied. We notice that Eq. (1) is only a nonresonant fire hose threshold and that parallel and oblique fire hose instabilities have thresholds generally lower than Eq. (1) because of the cyclotron resonances.

\subsection{Resonant interaction with particles}

Since these waves are left handed polarized and propagate nearly sunward in the solar wind plasma frame, back streaming ions which move upstream can not have resonant interaction with the waves, but those ions moving in the opposite direction relative to the wave propagation may. The resonant condition for such particles in the plasma rest frame is expressed as $\omega-\boldsymbol{k} \cdot \boldsymbol{V}=n \Omega_{p}$, where $\omega$ is the wave frequency, $\mathbf{V}$ is the proton velocity, $\Omega_{p}$ is the proton cyclotron frequency, and $n$ is an integer $(0, \pm 1, \pm 2 \ldots)$. For a wave and a particle relatively moving in opposite directions, 


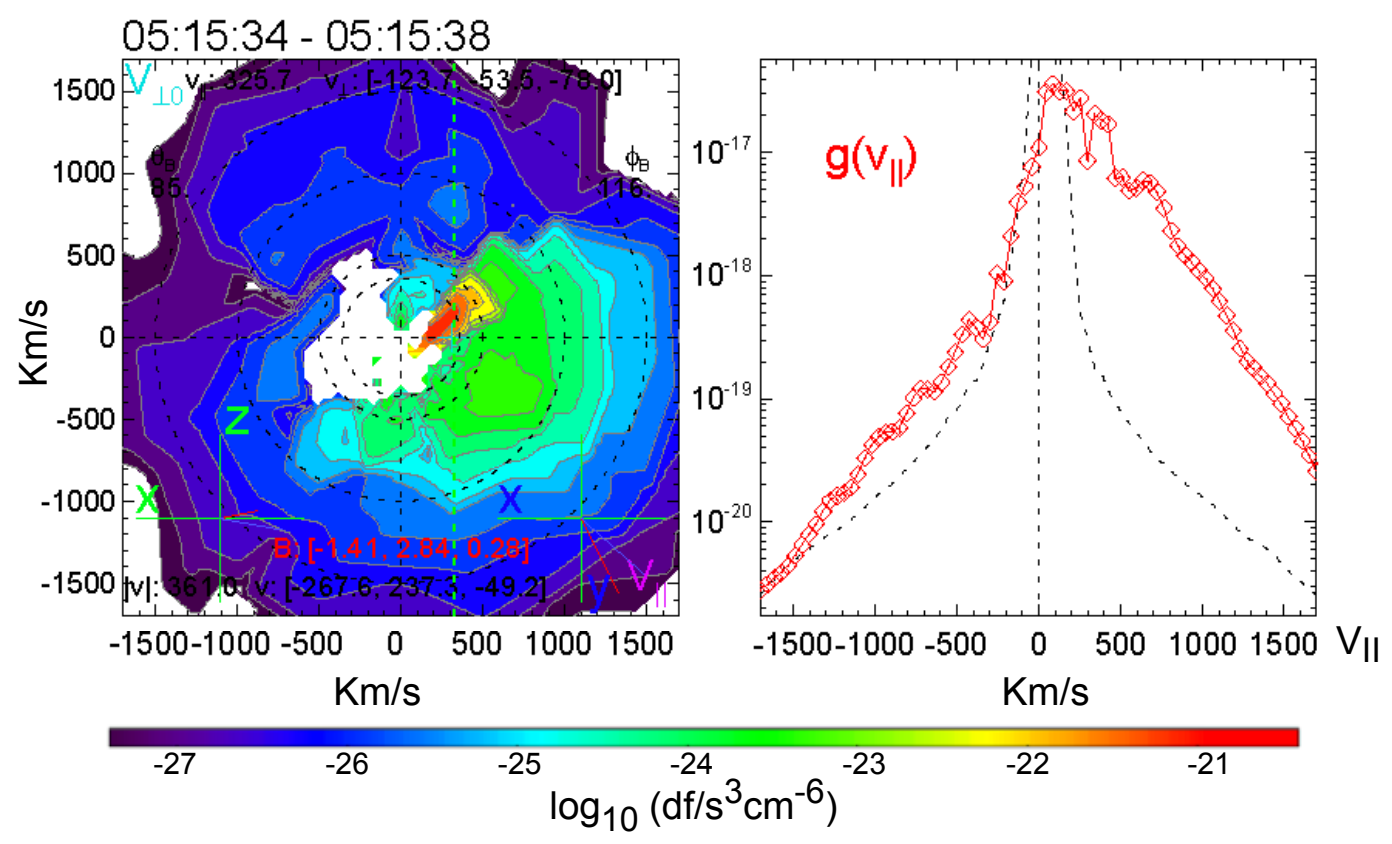

Fig. 6. The ion distribution during the interval A3. Left: a $\left(\mathbf{v}_{\|}, \mathbf{v}_{\perp}\right)$ slice through velocity space. Right: reduced distribution (a one-D cut along $v_{\|}$at $\left.v_{\perp}=0\right)$. The dotted curves are instrument one-count levels.

which Doppler shifts the wave frequency up to the proton gyrofrequency, the resonant condition can be written as $\omega+k_{\|} V_{\|}=\Omega_{p}$, where $k_{\|}$and $V_{\|}$are the components of the wave vector and proton velocity parallel to the ambient magnetic field, respectively. The energy of resonant protons can then be estimated with $V_{\| \mid}=\left(\Omega_{p}-\omega\right) / k_{\|}$. Taking the wave in period A3 as an example, using the data in Table 1, we have $k_{\|}=\left[2 \pi /\left(V_{\mathrm{obs}} \cdot T\right)\right] \cos \theta_{B k} \approx 0.0018 \mathrm{~km}^{-1}$, where the wave period $T=6 \mathrm{~s}$, and $\omega=\left(V_{0} / V_{\text {obs }}\right) 2 \pi / T \approx 0.23 \mathrm{~Hz}$, $\Omega_{p} \approx 0.80 \mathrm{~Hz}$. The velocity of the resonant protons $V_{\|}$is found to be $\sim 315 \mathrm{~km}$ (in the plasma frame), which corresponds to proton energy of $\sim 500 \mathrm{eV}$.

An effect of this resonant interaction is pitch angle scattering of particles, which diffuses the distribution around the resonant energy. Such interaction may also reduce the ion anisotropy and thus the free energy available for the firehose instability. In Fig. 6 we display the ion distribution during the interval A3. The left panel shows a $\left(\mathbf{v}_{\|}, \mathbf{v}_{\perp}\right)$ slice through velocity space, where $v_{\perp}$ is the component of the bulk flow perpendicular to $\mathbf{B}$. The right panel shows a reduced distribution, $g\left(v_{\|}\right)$. The distribution shows rather diffused ions with $V_{\|}$of several hundreds $(\sim 500-800) \mathrm{km} \mathrm{s}^{-1}$ in addition to the solar wind beam with a peak at $V_{\|} \sim 200-300 \mathrm{~km} \mathrm{~s}^{-1}$ (in the spacecraft frame). In the solar wind frame, these diffused ions would have $V_{\|}$of about $300-500 \mathrm{~km} \mathrm{~s}^{-1}$. We notice that from panel 2 of Fig. 5 the fire hose instability condition is relatively weak when electromagnetic fluctuations become large (intervals A3, A4, and B3). The resonant interaction described above may have played a role in reducing the ion temperature anisotropy.
Back streaming electrons may also have resonant interaction with the left handed wave propagating away from the bow shock in the solar wind rest frame. But for such interaction to occur, however, the streaming electrons' energy would need to be about $1 \mathrm{keV}$, but this was not observed.

\section{Conclusion}

We have analyzed in detail a period including three density holes in the foreshock region in terms of wave properties of the structures. We found that the electro-magnetic fluctuations associated with the occurrence of the density holes are consistent with left-hand polarized Alfvén waves propagating obliquely to the ambient magnetic field and away from the bow shock in the plasma frame. The wave amplitude grows non-linearly. The maximum amplitude developed coincides with the shock-like upstream edges of the density holes. Plasma conditions during the event seem to favor the growth of the firehose instability. Resonant interaction between the waves and ions streaming earthward at certain energy may occur, which could reduce the temperature anisotropy and stabilize the fluctuations.

Although the results obtained in this study represent a group of density hole events, they cannot be simply generalized to other density holes-like structure. The polarization sense may vary, which would change the roles of resonant interaction in the wave development. Also, there exist a group of density holes that are observed without SLAMS-like overshoot on the upstream edges of the structure (Wilber et al., 
2008), which is apparently not associated with growth of waves.

There are some important aspects of density holes which have not been addressed in this study. As we mentioned earlier, in-phase variations of the magnetic field strength and the plasma density are commonly observed, sometime with a high correlation. Such variations are a signature of fast mode oscillations. In these three events, however, such correlation is not obvious, and we find weak evidence for in-phase oscillations. But the compressional component of the fluctuations at similar frequency is obvious as seen in the variations in $|B|$ in Fig. 1. We do not rule out the possibility of the coexistence of fast mode waves in the fluctuations, which may play a role in causing large fluctuations of density. Also, the coexistence of the fast mode with the left hand polarization may suggest a coexistence of right hand parallel and left hand oblique fire hose instabilities. Other related important phenomena worth further study are occurrences of various high frequency waves associated with density holes (Parks et al., 2007), which include electromagnetic whistler mode waves and various electrostatic waves. Their occurrence may have important effects on the formation and the properties of the structure.

Acknowledgements. The research at UC Berkeley is performed under the auspices of a NASA Grant No. NNG04GF23G. Cluster is a joint project of ESA and NASA. The magnetic field data are obtained from the website of Cluster Active Archive. N. Lin would like to thank A. Teste for examination of electron data. N. Lin is very grateful to M. Kivelson, G. Le, and R. Lysak for helpful discussion. We also thank the two referees for their constructive comments and suggestions.

Topical Editor R. Nakamura thanks D. Constantinescu and another anonymous referee for their help in evaluating this paper.

\section{References}

Balogh, A., Dunlop, M. W., Cowley, S. W. H., et al.: The Cluster Magnetic Field Investigation, Space Sci. Rev., 79, 65-92, 1997.

Behlke, R., André, M., Buchert, S. C., Vaivads, A., Eriksson, A. I., Lucek, E. A., and Balogh, A.: Multi-point electric field measurements of Short Large-Amplitude Magnetic Structures (SLAMS) at the Earth's quasi-parallel bow shock, Geophys. Res. Lett., 30(4), 1177, doi:10.1029/2002GL015871, 2003.

Burgess, D.: What do we really know about upstream waves?, Adv. Space Res., 20, 673-682, 1997.

Daughton, W. and Gary, S. P.: Electromagnetic proton/proton instabilities in the solar wind, J. Geophys. Res., 103(A9), 20613 $20620,1998$.

Daughton, W., Gary, S. P., and Winske, D.: Electromagnetic proton/proton instabilities in the solar wind: Simulations, J. Geophys. Res., 104(A3), 4657-4667, 1999.

Eastwood, J. P., Balogh, A., Dunlop, M. W., Horbury, T. S., and Dandouras, I.: Cluster observations of fast magnetosonic waves in the terrestrial foreshock, Geophys. Res. Lett., 29(22), 2046, doi:10.1029/2002GL015582, 2002.
Eastwood, J. P., Balogh, A., Lucek, E. A., Mazelle, C., and Dandouras, I.: On the existence of Alfvén waves in the terrestrial foreshock, Ann. Geophys., 21, 1457-1465, 2003, http://www.ann-geophys.net/21/1457/2003/.

Eastwood, J. P., Lucek, E. A., Mazelle, C., Meziane, K., Narita, Y., Pickett, J., and Treumann, R. A.: The foreshock, Space Sci. Rev., 118, 41-94, 2005.

Gary, S. P., Li, H., O'Rourke, S., and Winske, D.: Proton resonant firehose instability: Temperature anisotropy and fluctuating field constraints, J. Geophys. Res., 103, 14 567-14 574, 1998.

Gustafsson, G., André, M., Carozzi, T., et al.: First results of electric field and density observations by Cluster EFW based on initial months of operation, Ann. Geophys., 19, 1219-1240, 2001, http://www.ann-geophys.net/19/1219/2001/.

Greenstadt, E. W., Le, G., and Strangeway, R. J.: ULF waves in the foreshock, Adv. Space Res., 15, 71-84, 1995.

Hellinger, P. and Matsumoto, H.: New kinetic instability: Oblique Alfvén fire hose, J. Geophys. Res., 105, 10 519-10 526, 2000.

Hellinger, P. and Matsumoto, H.: Nonlinear competition between the whistler and Alfvén fire hoses, J. Geophys. Res., 106, 13 215-13 218, 2001.

Hellinger, P. and Trávníček, P.: Parallel and oblique proton fire hose instabilities in the presence of alpha/proton drift: Hybrid simulations, J. Geophys. Res., 111, A01107, doi:10.1029/2005JA011318, 2006.

Hellinger, P., Trávníček, P., Kasper, J. C., and Lazarus, A. J.: Solar wind proton temperature anisotropy: Linear theory and WIND/SWE observations, Geophys. Res. Lett., 33, L09101, doi:10.1029/2006GL025925, 2006.

Kasper, J. C., Lazarus, A. J., and Gary, S. P.: Wind/SWE observations of firehose constraint on solar wind proton temperature anisotropy, Geophys. Res. Lett., 29(17), 1839, doi:10.1029/2002GL015128, 2002.

Lucek, E. A., Horbury, T. S., Dunlop, M. W., Cargill, P. J., Schwartz, S. J., Balogh, A., Brown, P., Carr, C., Fornacon, K.H., and Georgescu, E.: Cluster magnetic field observations at a quasi-parallel bow shock, Ann. Geophys., 20, 1699-1685, 2002, http://www.ann-geophys.net/20/1699/2002/.

Lucek, E. A., Horbury, T. S., Balogh, A., Dandouras, I., and Rème, H.: Cluster observations of hot flow anomalies, J. Geophys. Res., 109, A06207, doi:10.1029/2003JA010016, 2004.

Onsager, T. G., Thomsen, M. F., Gosling, J. T., and Bame, S. J.: Observational test of a hot flow anomaly formation mechanism, J. Geophys. Res., 95, 11 967-11974, 1990.

Parker, E. N.: Dynamical instability in an anisotropic ionized gas of low density, Phys. Rev., 109, 1874-1876, 1958.

Parks, G. K., Lee, E., Mozer, F., Wilber, M., Lucek, E., Dandouras, I., Rème, H., Mazelle, C., Cao, J. B., Meziane, K., Goldstein, M. L., and Escoubet, P.: Larmor radius size density holes discovered in the solar wind upstream of Earth's bow shock, Phys. Plasma, 13, 050701, doi:10.1063/1.2201056, 2006.

Parks, G. K., Lee, E., Lin, N., Mozer, F., Wilber, M., Lucek, E., Dandouras, I., Rème, H., Cao, J. B., Canu, P., CornilleauWehrlin, N., Décréau, P., Goldstein, M. L., and Escoubet, P.: Density holes in the upstream solar wind, in: Proceedings of The 6th Annual International Astrophysics Conference, edited by: Shaikh, D. and Zank, G. P., AIP, 9-15, 2007.

Paschman, G., Haerendel, G., Sckopke, N., Mobius, E., Luhr, H., and Carlson, C. W.: Three dimensional plasma structures with 
anomalous flow directions near the Earth's bow shock, J. Geophys. Res., 93, 11 279-11 294, 1988.

Rème, H., Bosqued, J. M., Sauvaud, J. A., et al.: The Cluster Ion Spectrometry (CIS) Experiment, Space Sci. Rev., 79, 303-350, 1997.

Russell, C. T. and Hoppe, M. M.: Upstream waves and particles, Space Sci. Rev., 34, 155-172, 1983.

Schwartz, S. J.: Shock and discontinuity normals, Mach numbers, and related parameters, in: Analysis Method for MultiSpacecraft Data, edited by: Paschmann, G. and Daly, P. W., ISSI/ESA, 249-270, 2000.

Schwartz, S. J., Chaloner, C. P., Hall, D. S., Christiansen, P. J., and Johnstones, A. D.: An active current sheet in the solar wind, Nature, 318, 269-271, 1985.

Schwartz, S. J. and Burgess, D.: Quasi-parallel shocks - A patchwork of three-dimensional structures, Geophys. Res. Lett., 18, 373-376, 1991.

Schwartz, S. J., Burgess, D., Wilkinson, W. P., Kessel, R. L., Dunlop, M., and Luhr, H.: Observations of short large-amplitude magnetic structures at a quasi-parallel shock, J. Geophys. Res., 97, 4209-4227, 1992.

Sentman, D. D., Edmiston, J. P., and Frank, L.: Instabilities of low frequency, parallel propagating electromagnetic waves in the Earth's foreshock region, J. Geophys. Res., 86, 7487-7497, 1981.

Sibeck, D. G., Borodkova, N. L., Zastenker, G. N., Romanov, S. A., and Sauvaud, J.-A.: Gross deformation of the dayside magnetopause, Geophys. Res. Lett., 25, 453-456, 1998.
Sibeck, D. G., Phan, T.-D., Lin, R., Lepping, R. P., and Szabo, A.: Wind observations of foreshock cavities: A case study, J. Geophys. Res., 107(A10), 1271, doi:10.1029/2001JA007539, 2002.

Stasiewicz, K., Longmore, M., Buchert, S., Shukla, P. K., Lavraud, B., and Pickett, J.: Properties of fast magnetosonic shocklets at the bow shock, Geophys. Res. Lett., 30, SSC4-1, doi:10.1029/2003GL017971, 2003.

Thomsen, M. F., Gosling, J. T., Fuselier, S. A., Bame, S. J., and Russell, C. T.: Hot, diamagnetic cavities near the Earth's bow shock, J. Geophys. Res., 91, 2961-2973, 1986.

Thomsen, M. F., Gosling, J. T., Bame, S. J., and Russell, C. T.: Magnetic pulsations at the quasi-parallel shock, J. Geophys. Res., 95, 957-966, 1990.

Treumann, R. A. and Baumjohann, W.: Advanced Space Plasma Physics, Imperial College Press, London, 51-55, 1997.

Wilber, M., Parks, G. K., Meziane, K., Lin, N., Lee, E., Mazelle, C., and Harris, A.: Foreshock density holes in the context of known upstream plasma structures, Ann. Geophys., in press, 2008.

Wilkinson, W. P.: The Earth's quasi-parallel bow shock: Review of observations and perspectives for Cluster, Planet. Space Sci., 51, 629-647, 2003.

Yoon, P. H.: Electromagnetic fire hose instability in a full relativistic bi-Maxwellian plasma, Phys. Fluids B, 2, 842-844, 1990. 